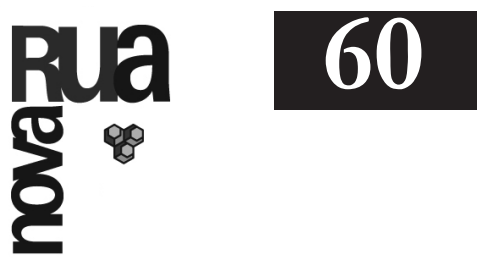

\title{
COMPROMISO Y APOYO ORGANIZACIONAL en docentes de la Universidad Autónoma de Ciudad Juárez campus sur
}

Mariana Ivonne Nava Soto Karla Yolanda Ruiz Martinez Emmanuel Francisco García Uribe Universidad Autónoma de Ciudad Juárez

Recibido: 26 de agosto de 2015. Aceptado: 25 de noviembre de 2015

\section{RESUMEN}

I compromiso organiza4 cional es un estado psicológico que determina la relación del empleado con la organización e influye en la decisión de continuar en la organización. Se ha comprobado que el Apoyo Organizacional Percibido es un antecedente importante del compromiso organizacional.

El objetivo de esta investigación es conocer la percepción de apoyo y compromiso organizacional de docentes de la Universidad Autónoma de Ciudad Juárez campus sur con respecto a la percepción de apoyo y compromiso organizacional. La muestra se constituyó por 188 docentes. Los resultados mostraron diferencia significativa en la dimensión de compromiso afectivo y normativo en docentes de los institutos de ICSA e ICB. Con respecto a la percepción del apoyo organizacional no se encontró diferencia significativa entre los docentes de los distintos institutos. En general los docentes presentaron un nivel medio de compromiso organizacional. 
Palabras clave: Compromiso organizacional, apoyo organizacional, docentes.

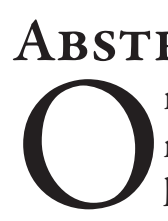

rganizational commitment is a psychological state that determines the relationship between the employee and the organization and it influences the decision to continue in the organization. It has been proved that the perceived organizational support is an important precedent of the organizational commitment.

The object of this investigation is to know the perception of support and organizational commitment about the teachers of Universidad Autónoma de Ciudad Juárez Campus Sur. The sample was made up by 188 teachers. The results showed a significant difference in the dimension of affective and normative commitment at ICSA and ICB institutes. Regarding to the perception of organizational support it was not found a significant difference between teachers from the institutes involved. The teachers presented a medium level of organizational commitment generally.
Key words: Organizational commitment, organizational support, teachers.

\section{Planteamiento DEL PROBLEMA}

El campo del comportamiento organizacional ha estudiado una serie de actitudes que suelen tener los empleados hacia el trabajo (Robbins \& Judge, 2009), esto se debe a que determinan en gran medida como sus integrantes perciben su ambiente de trabajo, se comprometen con las metas y objetivos organizacionales (Torres \& Díaz, 2012).

Una de las actitudes más importantes y que ha sido debatida ampliamente por académicos y profesionales es el compromiso organizacional, que es definido como una fuerza relativa de la identificación de un individuo con una organización (Wu \& Liu, 2014).

La importancia del estudio del compromiso organizacional radica en los efectos que implica para la organización (Ayensa \& González, 2007); un bajo nivel de compromiso en los empleados trae consigo la reducción de la eficacia organizacional (Soberanes \& De la Fuente, 2009), seguido por los costos de rotación de personal que se generan a causa de la decisión del empleado de buscar otro empleo (Arias, 2001) así como el incumplimiento de la metas organizacionales (Maldonado, Guillén, \& Carranza, 2011).

Cabe destacar que el compromiso de los empleados puede presentarse con distinta intensidad; puede ir desde el mínimo compromiso con el empleador hasta un intenso compromiso (Robles \& De la Garza, 2009). En torno a esto se han realizado diversos estudios intentando descubrir aquellos factores que influyen en la intensidad de la dedicación de los empleados en las organizaciones y existen hallazgos que apuntan hacia la Percepción del Apoyo Organizacional (PAO) como un antecedente importante del compromiso organizacional (Eisenberger, Huntington, Hutchison, \& Sowa, 1986).

Dichos resultados muestran que la percepción del empleado acerca del compromiso de la organización hacia él, contribuye después a formar el compromiso del empleado 
con la organización (Moideenkutty et. al., 2001).

Asimismo, se han realizado estudios analizando el compromiso organizacional y $\mathrm{PAO}$, en los que se ha demostrado su importancia. En una revisión de más de 70 estudios relativos a PAO y mediante un metanálisi se pudo observar que tiene relación favorable para los trabajadores y la organización, lo cual genera beneficios en cuanto al compromiso organizacional con mayor presencia en su dimensión afectiva, el rendimiento y disminución de la intención de abandono (Rhoades \& Eisenberger, 2002).

A pesar de su importancia, los estudios que contemplan el análisis de la percepción, el apoyo organizacional y el compromiso organizacional, ha sido escaso el estudio del primero en nuestro país, y el segundo particularmente en el ámbito educativo. Esto constituye una limitante, ya que imposibilita el originar acciones que contribuyan a desarrollar el compromiso organizacional en el docente (Barraza \& Acosta, 2008). Por su parte, la Organización de las $\mathrm{Na}$ ciones Unidas para la Educa- ción, la Ciencia y la Cultura (UNESCO) ha sugerido que mediante el compromiso organizacional se puede lograr el desarrollo y la mejora de la educación, por lo que sugiere un análisis profundo y reflexivo (Hernández \& Guerrero, 2006).

De acuerdo con lo argumentado y dados los efectos negativos que implica para las organizaciones no contar con empleados comprometidos, este estudio se enfoca en establecer, en una muestra del personal docente del campus sur de la Universidad Autónoma de Ciudad Juárez, si existen diferencias en el compromiso organizacional y la percepción del apoyo organizacional, teniendo como referencia el modelo multidimensional de compromiso organizacional (Meyer \& Allen, 1991) y la definición de percepción de apoyo organizacional.

\section{Pregunta de IN- VESTIGACIÓN}

- Existe una diferencia significativa entre el com6 promiso organizacional y la percepción de apoyo entre los docentes de la Universidad Autónoma de Ciudad Juárez campus sur, en función del instituto al que pertenece el programa académico al que están adscritos?

\section{Objetivo \\ GENERAL \\ dentificar si existe una
diferencia
entre el compromificativa} ganizacional y la percepción de apoyo entre los docentes de la Universidad Autónoma de Ciudad Juárez campus sur, en función del instituto al que pertenece el programa académico al que están adscritos.

\section{Hipótesis}

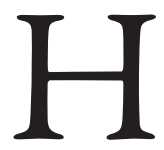

$\left\{\begin{array}{l}\text { 1: Existe una dife- } \\ \text { rencia significativa } \\ \text { entre el compromiso }\end{array}\right.$ organizacional y la percepción de apoyo entre los docentes de la Universidad Autónoma de Ciudad Juárez campus sur, en función del instituto al que pertenece el programa académico al que están adscritos.

H2: No existe una diferencia significativa entre el compromiso organizacional y la percepción de apoyo entre los docentes de la Universidad Autónoma de Ciudad Juárez campus sur, en función del instituto al que pertenece el pro- 
grama académico al que están adscritos.

\section{JUSTIFICACIÓN

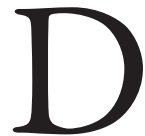 ebido a la escasa pre- sencia de estudios so- bre el tema abordado} en el ámbito educativo y tomando en cuenta que la educación es un factor esencial para la formación de profesionistas en el país, es de suma importancia enfocar el estudio en este contexto y notablemente conocer si existe una diferencia significativa entre ambos grupos siendo empleados de la misma organización.

Este estudio permitirá conocer si existen diferencias entre ambos grupos esta información servirá como apoyo al departamento de recursos humanos ya que también se podrá obtener información acerca del nivel de compromiso y percepción del apoyo organizacional de los docentes, adicionalmente se podrá tener un punto de partida para generar estrategias que permitan fomentar, mantener, aumentar o en su caso alinear el nivel de estas dos actitudes en el trabajo.

Los beneficios que se obtienen al generar específicamente el compromiso organi- zacional, son altos niveles de rentabilidad (Yasar, Emhan, \& Ebere, 2014), un mejor esfuerzo del empleado, genera la intención de permanencia, lo que a su vez implica reducción de costos por rotación de personal (Arias, 2001), y no requieren ser supervisados debido a que toman las metas y objetivos organizacionales como propios (Soberanes \& De la Fuente, 2009).

\section{REVISIÓN DE LITERATURA}

\section{Compromiso Organizacio-} nal

1 nizacional se define identificación y participación que adopta un individuo hacia una determinada organización (Porter, Steers, Mowday, \& Boulian, 1974).

Sin embargo, la definición tradicional del Compromiso Organizacional se refiere al concepto aportado por Meyer y Allen (1991), que lo consideran como un estado psicológico que determina la relación del empleado con la organización e implica la toma de decisión por el empleado de continuar en la misma.

Ruiz de Alba (2013) menciona que "El objeto del compromiso puede ser hacia una persona, una institución o una meta. No tienen por qué ser excluyentes los objetos del compromiso, pero lógicamente van a variar respecto a la naturaleza del vínculo generado".

El compromiso organizacional generado en el empleado deduce el comportamiento en su lugar de trabajo contribuyendo al cumplimiento de las metas organizacionales (Maldonado, Guillén, \& Carranza, 2012), es decir, el empleado toma en cuenta todos los beneficios positivos recibidos por parte de la organización para su bienestar laboral, que consecuentemente lo induce a generar un sentimiento de compromiso de su trabajo hacia la institución (Florez, 2014).

Se señala que un fuerte grado de compromiso organizacional se representa en base al apoyo y aceptación de las metas y valores de la organización, la habilidad de aportar un esfuerzo significativo que ayude a beneficiar a la organización y el deseo de permane- 
cer en ella (Mowday, Steers, \& Porter, 1979), incluso conocer el grado de compromiso organizacional es de utilidad para predecir si se desarrollará rotación y ausentismo (Ko, Price, \& Mueller, 1997).

Mowday, Steers, \& Porter (1979) deducen que el compromiso organizacional es un modelo de constructo unidimensional que representa un estado en el que un individuo se identifica con una determinada organización y sus objetivos, así como el deseo de mantener su membrecía a fin de facilitar estos objetivos. El utilizar este constructo permite evaluar las diferentes perspectivas que existen en el empleado al comprometerse con la organización (Frutos, Ruiz, \& San Martin, 1998).

Sin embargo, el modelo elaborado por Meyer \& Allen (1991) es el más apropiado para poder conocer las perspectivas, ya que este modelo está conformado por tres dimensiones que son: el apego afectivo con la organización, los costos percibidos que se asocian con el abandono de la organización y la obligación de permanecer con la organización, las cuales se deno- minan como Compromiso afectivo (CA), compromiso de continuidad (CC) y compromiso normativo $(\mathrm{CN})$, respectivamente.

Desde la teoría del vínculo afectivo, el compromiso es definido como un apego emocional entre el empleado con la organización, identificación y participación en sus objetivos y valores, que se caracteriza por tres factores: (a) identificación al adoptar como propias las metas y valores de la organización, (b) esfuerzo o implicación en las actividades de rol de trabajo del mismo, y (c) la lealtad, un sentimiento de afecto y apego a la organización (Buchanan, 1974; Porter, Steers, Mowday, \& Boulian, 1974).

Esta dimensión se denomina como afectiva. Los empleados que desarrollan este tipo de compromiso adoptan el sentimiento de orgullo al pertenecer a la organización en la que laboran (Florez, 2014). Por ende, Zamora (2009) argumenta que los sujetos que cuentan con un grado elevado de compromiso afectivo permanecen en la organización porque sienten que "desean hacerlo".
Según Becker (1960), con base en la "Teoría del intercambio social" se define al compromiso organizacional como una relación que se funda entre el empleado con la organización, como efecto de pequeñas inversiones (sidebets) llevadas a cabo en el tiempo que han permanecido en la organización, de tal manera que, si la organización le brinda acciones o atenciones que beneficien al empleado, se establece un compromiso denominado como dimensión de continuidad. A lo que Betanzos, Andrade, \& Paz (2006) sugieren que desde esta teoría, se adopta un compromiso de continuidad en resultado de una transacción de contribuciones entre el empleado a través de beneficios e incentivos recibidos por parte de su empresa. Se dice que los empleados con un alto grado de compromiso de continuidad permanecen a la organización debido a que "lo necesitan" (Zamora, 2009).

Con respecto a la norma de la reciprocidad, Gouldner (1960) aporta que aquel individuo que recibe algún beneficio se ve obligado moralmente a retribuir al emisor. Confor- 
me a esta teoría, se desarrolla el compromiso a través de las recompensas otorgadas por parte de la organización hacia el empleado, lo que inculca un sentido de obligación moral de corresponder (Ko, Price, \& Mueller, 1997), es decir, el empleado que con el tiempo desarrolla un amplio sentimiento que obliga su permanencia, a través de adoptar la sensación de deuda hacia alguna oportunidad u recompensa obtenida por parte de la organización, conllevando al empleado a valorarlo (Barraza \& Acosta, 2008).

Sin embargo, las inversiones por parte de la organización pueden generar un desequilibrio en la relación del empleado con la organización y hacer que los empleados sientan la obligación de corresponder, comprometiéndose hasta que la deuda quede saldada (Meyer \& Allen, 1991). Esta dimensión es denominada normativa.

El compromiso normativo conlleva que el empleado cumpla sus obligaciones en la organización no simplemente por que lo tiene que hacer, sino porque cree que es lo correcto, un empleado que desa- rrolla compromiso normativo se enfoca a cumplir con las metas de la organización, pero también se responsabiliza hacia los demás para ser aprobado ante la sociedad (Betanzos, Andrade, \& Paz, 2006). Es decir, un empleado con alto grado de compromiso normativo permanece en la organización porque siente que "debe hacerlo" (Zamora, 2009).

Debido a su importancia, el compromiso organizacional recientemente ha recibido atención considerable, no solo de investigadores organizacionales sino también de investigadores de la educación, los cuales han realizado un creciente número de estudios encaminados a identificar antecedentes y resultados del compromiso organizacional (Çağla, 2013), así como los factores que aumentan el compromiso organizacional.

El compromiso organizacional en los docentes tiene influencia en la distribución de poder y responsabilidad, que los lleva a utilizar su talento, capacidades de fomentar creatividad e inversión en el aprendizaje, que desde una perspectiva universitaria es de suma importancia el poten- ciar la necesidad de contribuir significativamente en su labor (García de Pilo, Useche, \& Schelesinger, 2013).

Por su parte, la institución se favorece a través de empleados altamente comprometidos con la institución a la que pertenecen, manteniendo una ventaja competitiva con las capacidades con las que cuentan su personal educativo (Edel, García, \& Casiano, 2007).

\section{Percepción Del APOYO ORGANIZACIONAL}

La percepción del apoyo organizacional es el constructo central en la teoría de apoyo organizacional (Baran, Rhoades, \& Miller, 2012), la cual surgió estudiando el compromiso de los empleados, específicamente si los gerentes influyen en el compromiso de los empleados hacia la organización. Dicha teoría fue desarrollada por Eisenberger et.al., (1986), y plantea que los empleados desarrollan creencias globales acerca de cómo la organización valora sus contribuciones y cuida de su bienestar.

Para su desarrollo se tomó como punto de partida lo su- 
gerido por Levinson (1965), según este autor los empleados tienden a ver las acciones realizadas por agentes individuales como actos realizados por la organización, es decir, se realiza una personificación de la organización, por lo tanto aplicado en la teoría de apoyo organizacional, el empleado al desarrollar creencias globales acerca de cómo la organización valora sus contribuciones y cuida de su bienestar, lo hace a partir de una personificación de la organización.

Por otra parte, se dice que la teoría del apoyo organizacional es también una aplicación de la teoría de intercambio social en cuanto a la relación empleador-empleado (Baran, Rhoades, \& Miller, 2012). Esta teoría fue desarrollada para explicar el inicio, el fortalecimiento y el mantenimiento continuo de las relaciones interpersonales Eisenberger $e t$. al. (1997).

Sin embargo, la teoría del intercambio social aplicada en el contexto del trabajo sostiene que los empleados están dispuestos a intercambiar su esfuerzo y tiempo por varias recompensas que ofrece la organización; postula que los empleados están dispuestos a intercambiar el rendimiento laboral por factores adicionales que son menos tangibles, tales como sentirse valorados y apoyados (Sivalogathasan \& Ashmel, 2014).

El centro de la teoría de intercambio social es la norma de reciprocidad, la cual consiste en devolver los beneficios recibidos (Gouldner, 1960). En el caso de la percepción del apoyo organizacional se argumenta que produce un sentido de obligación en el empleado que posteriormente lo lleva a buscar la forma de ayudar a alcanzar los objetivos organizacionales (Eisenberg, Armeli, Rexwinkel, Lynch, \& Rhoades, 2001), es de esta forma como el empleado devuelve los beneficios recibidos.

La percepción del apoyo organizacional puede ser influenciada por diversos aspectos del tratamiento que se le da al empleado y, a su vez, influye la interpretación que adopta el empleado con respecto al trato organizacional que se le ofrece (Robbins \& Judge, 2009); en esta situación los empleados suelen sentir este apoyo porque son empleados valiosos para sus organizaciones (Colakoglu, Culha, \& Atay, 2010).

Por otra parte, mediante una extensa revisión de estudios que incluyen el análisis de la variable $\mathrm{PAO}$, se logró identificar como principales consecuencias un incremento en el desempeño, reduce el intento de abandono, y aumenta el compromiso afectivo, esta última en cuanto a su relación, se mostró fuerte y positiva (Rhoades \& Eisenberger, 2002).

Debido al efecto que se ha demostrado sobre el compromiso organizacional, se han realizado diversos estudios que contemplan el análisis tanto de apoyo organizacional como compromiso organizacional, entre los que ha encontrado que el compromiso organizacional mejora debido a la percepción de apoyo organizacional y que genera en los empleados motivación ante el trabajo desafiante (Shumaila, Aslam, Sadaqat, Maqsood, \& Sadaf, 2012), el apoyo aumenta el compromiso y es esto lo que a su vez aumenta las intenciones de compartir conocimiento (Tormo \& Osca, 2011), los empleados desarrollan un sentido de apego a la organización en gran medida 


\section{7 novage}

sobre la base de la percepción acerca del apoyo que proporciona la organización (Balfour \& Wechsler, 1991). Asimismo, Arias (2001) obtuvo como resultado que el factor más importante para aumentar el compromiso organizacional es el apoyo organizacional percibido.

Con respecto al campo educativo Litlewood (2003) en su estudio estableció como objetivo determinar la relación de PAO y el compromiso organizacional. Los resultados mostraron que la PAO se relaciona con el compromiso afectivo y normativo pero no con la dimensión de continuidad, lo que sugiere que los empleados no asocian la preocupación de la organización por su bienestar con los costos que implicarían abandonarla.

Los resultados de estos estudios apuntan a lo señalado en estudios anteriores que han mostrado al apoyo organizacional como un factor importante para aumentar el compromiso organizacional (Rhoades \& Eisenberger, 2002).

\section{Metodología} 1 presente estudio es
descriptivo-comparati-
vo, ya que se busca medir las dimensiones del fenómeno a estudiar y describir las diferencias de variables en dos o más grupos (Müggenburg \& Pérez, 2007); es de tipo transversal con diseño no experimental (Hernández et al., 1991). Se pretende comprobar si existe una diferencia significativa en el compromiso organizacional y percepción de apoyo organizacional entre docentes del campus sur por instituto. Los institutos son: Instituto de Ciencias Sociales y Administración (ICSA), Instituto de Ingeniería y Tecnología (IIT), Instituto de Arquitectura, Diseño y Arte (IADA), Instituto de Ciencias Biomédicas (ICB). Para efectos de este estudio no se considera el tipo de contrato de los docentes, ni características demográficas.

El universo de estudio está constituido por docentes que laboran en la Universidad Autónoma de Ciudad Juárez campus sur, localizada en el estado de Chihuahua que se encuentra en la región norte de la República Mexicana.
La población consta de 367 docentes de donde se obtiene una muestra de 188 docentes, con un nivel de confianza del 95\% con un margen de error de $5 \%$. Una vez obtenida la muestra se realizó una estratificación por instituto. 


\section{Rua 68}

Tabla 1. Estratificación de la muestra por institutos

\begin{tabular}{|l|l|l|}
\hline INSTITUTOS & TOTAL DOCENTES & MUESTRA ESTRATIFICADA \\
\hline ICSA-CU & 165 & 85 \\
\hline IADA-CU & 37 & 19 \\
\hline ICB-CU & 116 & 59 \\
\hline IIT-CU & 49 & 25 \\
\hline TOTAL & 367 & 188 \\
\hline
\end{tabular}

El instrumento a utilizar es el desarrollado por Meyer y Allen (1991), en este se miden tres dimensiones: compromiso afectivo, compromiso normativo y compromiso de continuidad; se encuentra conformado por 18 items. Para medir la percepción del apoyo organizacional se utilizará el cuestionario desarrollado por Eisenberger $e$ t. al., (1986), este constructo es unidimensional y se conforma por 8 items en su versión corta.

\section{ANÁlisis DE DATOS}

Ce utilizó el paquete Statistical Package for the Social Sciences (SPSS) para realizar el análisis ANOVA para datos independientes. Para obtener la identificación de los niveles de compromiso organizacional se construyó un baremo de intensidad considerando las puntuaciones mínimas y máximas posibles a obtener de acuerdo al número de items de cada cuestionario.

\section{Resultados}

D n la tabla 2 se puede 4 observar que no existe diferencia significativa entre docentes de los distintos institutos con respecto a la percepción del apoyo organizacional, sin embargo, el nivel de intensidad de apoyo percibido por institutos se encuentra en un nivel medio según muestran los resultados de la tabla 3. 
Tabla 2. ANOVA de un factor.

\begin{tabular}{|l|l|l|l|l|l|}
\hline \multicolumn{1}{|c|}{ Total de apoyo organizacional percibido } \\
\hline & Suma de cuadrados & gl & Media cuadrática & F & Sig. \\
\hline Inter-grupos & 146,427 & 3 & 48,809 &, 672 &, 570 \\
\hline Intra-grupos & 11036,163 & 152 & 72,606 & & \\
\hline Total & 11182,590 & 155 & & & \\
\hline
\end{tabular}

Tabla 3. Nivel de intensidad de apoyo organizacional percibido por instituto

\begin{tabular}{|l|l|l|l|l|l|}
\hline \multicolumn{2}{|c|}{} & \multicolumn{4}{|l|}{ Instituto } \\
\cline { 2 - 6 } \multicolumn{2}{|c|}{} & ICSA & ICB & IADA & ITT \\
\hline \multirow{2}{*}{\begin{tabular}{l} 
Nivel de apoyo $\begin{array}{l}\text { organizaciona } \\
\text { percibido }\end{array}$ \\
\cline { 2 - 7 }
\end{tabular}} & Recuento & Recuento & Recuento & Recuento & Recuento \\
\cline { 2 - 7 } & Bajo & 4 & 1 & 2 & 3 \\
\cline { 2 - 7 } & Medio & 57 & 25 & 12 & 13 \\
\cline { 2 - 6 } & Alto & 23 & 4 & 4 & 8 \\
\hline
\end{tabular}

Con respecto a la dimensión de compromiso afectivo los resultados de la tabla 4 muestran que existe una diferencia significativa entre los grupos analizados, posteriormente en la tabla 5 de comparaciones múltiples, se puede observar que dicha diferencia se presenta entre los institutos de ICSA e ICB. 
Tabla 4. ANOVA de un factor compromiso afectivo

\begin{tabular}{|l|l|l|l|l|l|}
\hline \multicolumn{7}{|c|}{ Total de compromiso afectivo } \\
\hline & $\begin{array}{l}\text { Suma de } \\
\text { cuadrados }\end{array}$ & Gl & Media cuadrática & F & Sig. \\
\hline Inter-grupos & 1065,691 & 3 & 355,230 & 7,012 &, 000 \\
\hline Intra-grupos & 7751,073 & 153 & 50,661 & & \\
\hline Total & 8816,764 & 156 & & & \\
\hline
\end{tabular}

Tabla 5. Comparaciones múltiples compromiso afectivo

\begin{tabular}{|l|l|l|l|l|l|l|}
\hline \multicolumn{7}{|c|}{ Variable dependiente: Total de compromiso afectivo Scheffé } \\
\hline (I) Instituto & (J) Instituto & $\begin{array}{l}\text { Diferencia de } \\
\text { medias (I-J) }\end{array}$ & Error típico & Sig. & $\begin{array}{l}\text { Intervalo de } \\
\text { confianza al } \\
95 \%\end{array}$ & \\
\hline & & & & & $\begin{array}{l}\text { Límite } \\
\text { inferior }\end{array}$ & $\begin{array}{l}\text { Límite } \\
\text { superior }\end{array}$ \\
\hline ICSA & ICB & $6,534^{*}$ & 1,533 &, 001 & 2,20 & 10,87 \\
\hline & IADA & 4,447 & 1,808 &, 114 &,- 66 & 9,56 \\
\hline & IIT & 3,060 & 1,622 &, 317 & $-1,52$ & 7,64 \\
\hline ICB & ICSA & $-6,534^{*}$ & 1,533 &, 001 & $-10,87$ & $-2,20$ \\
\hline & IADA & $-2,087$ & 2,101 &, 804 & $-8,03$ & 3,85 \\
\hline & IIT & $-3,474$ & 1,943 &, 365 & $-8,97$ & 2,02 \\
\hline IADA & ICSA & $-4,447$ & 1,808 &, 114 & $-9,56$ &, 66 \\
\hline & ICB & 2,087 & 2,101 &, 804 & $-3,85$ & 8,03 \\
\hline & IIT & $-1,387$ & 2,166 &, 938 & $-7,51$ & 4,74 \\
\hline IIT & ICSA & $-3,060$ & 1,622 &, 317 & $-7,64$ & 1,52 \\
\hline & ICB & 3,474 & 1,943 &, 365 & $-2,02$ & 8,97 \\
\hline & IADA & 1,387 & 2,166 &, 938 & $-4,74$ & 7,51 \\
\hline
\end{tabular}


Tabla 6. ANOVA de un factor compromiso de continuidad.

\begin{tabular}{|l|l|l|l|l|l|}
\hline \multicolumn{7}{|c|}{ Total de compromiso de continuidad } \\
\hline & $\begin{array}{l}\text { Suma de } \\
\text { cuadrados }\end{array}$ & gl & Media cuadrática & F & Sig. \\
\hline Inter-grupos & 90,852 & 3 & 30,284 &, 614 &, 607 \\
\hline Intra-grupos & 7549,938 & 153 & 49,346 & & \\
\hline Total & 7640,790 & 156 & & & \\
\hline
\end{tabular}

Tabla 7. ANOVA de un factor compromiso normativo

\begin{tabular}{|l|l|l|l|l|l|}
\hline \multicolumn{7}{|c|}{ Total de compromiso normativo } \\
\hline & $\begin{array}{l}\text { Suma de } \\
\text { cuadrados }\end{array}$ & gl & $\begin{array}{l}\text { Media } \\
\text { cuadrática }\end{array}$ & F & Sig. \\
\hline Inter-grupos & 639,435 & 3 & 213,145 & 4,292 &, 006 \\
\hline Intra-grupos & 7598,297 & 153 & 49,662 & & \\
\hline Total & 8237,732 & 156 & & & \\
\hline
\end{tabular}

En relación a la dimensión que corresponde al compromiso de continuidad no se obtuvo diferencia significativa entre las medias de los grupos analizados como muestra la tabla 6.

Posteriormente los resultados mostraron con respecto a la dimensión de compromiso normativo que se presentó diferencia significativa en las medias analizadas, lo cual se puede observar en la tabla 7 . La diferencia se presentó de igual forma en los institutos de ICSA e ICB, como se puede observar en la Tabla 8. 
Tabla 8. Comparaciones múltiples compromiso normativo

\begin{tabular}{|c|c|c|c|c|c|c|}
\hline \multicolumn{7}{|c|}{ Variable dependiente: Total de compromiso normativo Scheffé } \\
\hline (I) Instituto & (J) Instituto & $\begin{array}{c}\text { Diferencia } \\
\text { de medias (I-J) }\end{array}$ & Error típico & Sig. & \multicolumn{2}{c|}{ Intervalo de confianza al 95\% } \\
\cline { 5 - 7 } & & & & & $\begin{array}{c}\text { Límite } \\
\text { inferior }\end{array}$ & $\begin{array}{c}\text { Límite su- } \\
\text { perior }\end{array}$ \\
\hline ICSA & ICB & $4,531^{*}$ & 1,499 &, 031 &, 29 & 8,77 \\
\hline & IADA & 4,353 & 1,830 &, 134 &,- 82 & 9,53 \\
\hline & IIT & 2,824 & 1,606 &, 380 & $-1,71$ & 7,36 \\
\hline ICB & ICSA & $-4,531^{*}$ & 1,499 &, 031 & $-8,77$ &,- 29 \\
\hline & IADA &,- 178 & 2,101 & 1,000 & $-6,12$ & 5,76 \\
\hline IADA & IIT & $-1,707$ & 1,908 &, 849 & $-7,10$ & 3,69 \\
\hline & ICSA & $-4,353$ & 1,830 &, 134 & $-9,53$ &, 82 \\
\hline & ICB &, 178 & 2,101 & 1,000 & $-5,76$ & 6,12 \\
\hline IIT & IIT & $-1,529$ & 2,178 &, 920 & $-7,69$ & 4,63 \\
\hline & ICSA & $-2,824$ & 1,606 &, 380 & $-7,36$ & 1,71 \\
\hline & ICB & 1,707 & 1,908 &, 849 & $-3,69$ & 7,10 \\
\hline$*$. La diferencia de medias es significativa al nivel 0.05. & 2,178 &, 920 & $-4,63$ & 7,69 \\
\hline
\end{tabular}

Comparando las medias obtenidas con respecto al compromiso organizacional de forma global, se obtuvo que se rechaza la hipótesis nula, ya que existe diferencia significativa entre los grupos analizados; los resultados se muestran en la tabla 9. La tabla 10 muestra que dicha diferencia se presentó entre los institutos de ICSA e ICB. 
Tabla 9. ANOVA de un factor total de compromiso organizacional

\begin{tabular}{|l|l|l|l|l|l|}
\hline \multicolumn{7}{|c|}{ Total de compromiso organizacional } \\
\hline & $\begin{array}{l}\text { Suma de } \\
\text { cuadrados }\end{array}$ & gl & Media cuadrática & F & Sig. \\
\hline Inter-grupos & 5145,690 & 3 & 1715,230 & 6,945 &, 000 \\
\hline Intra-grupos & 37290,981 & 151 & 246,960 & & \\
\hline Total & 42436,671 & 154 & & & \\
\hline
\end{tabular}

Tabla 10. Comparaciones múltiples total de compromiso organizacional

\begin{tabular}{|c|c|c|c|c|c|c|}
\hline \multicolumn{7}{|c|}{$\begin{array}{l}\text { Variable dependiente: Total de compromiso organizacional } \\
\text { Scheffé }\end{array}$} \\
\hline \multirow[t]{2}{*}{ (I) Instituto } & \multirow{2}{*}{$\begin{array}{l}(\mathrm{J}) \text { Insti- } \\
\text { tuto }\end{array}$} & \multirow{2}{*}{$\begin{array}{l}\text { Diferencia } \\
\text { de medias (I-J) }\end{array}$} & \multirow[t]{2}{*}{ Error típico } & \multirow[t]{2}{*}{ Sig. } & \multicolumn{2}{|c|}{ Intervalo de confianza al 95\% } \\
\hline & & & & & $\begin{array}{l}\text { Límite in- } \\
\text { ferior }\end{array}$ & $\begin{array}{l}\text { Límite su- } \\
\text { perior }\end{array}$ \\
\hline \multirow[t]{3}{*}{ ICSA } & ICB & $13,893^{*}$ & 3,429 & 001 & 4,20 & 23,59 \\
\hline & IADA & 11,536 & 4,082 &, 050 & ,00 & 23,08 \\
\hline & IIT & 6,596 & 3,580 & ,338 & $-3,53$ & 16,72 \\
\hline \multirow[t]{3}{*}{ ICB } & ICSA & $-13,893^{*}$ & 3,429 &, 001 & $-23,59$ & $-4,20$ \\
\hline & IADA & $-2,357$ & 4,748 & 970 & $-15,78$ & 11,07 \\
\hline & IIT & $-7,297$ & 4,324 &, 418 & $-19,52$ & 4,93 \\
\hline \multirow[t]{3}{*}{ IADA } & ICSA & $-11,536$ & 4,082 &, 050 & $-23,08$ & 00 \\
\hline & $\mathrm{ICB}$ & 2,357 & 4,748 & 970 & $-11,07$ & 15,78 \\
\hline & IIT & $-4,940$ & 4,858 & ,793 & $-18,67$ & 8,79 \\
\hline \multirow[t]{3}{*}{ IIT } & ICSA & $-6,596$ & 3,580 & ,338 & $-16,72$ & 3,53 \\
\hline & ICB & 7,297 & 4,324 & ,418 & $-4,93$ & 19,52 \\
\hline & IADA & 4,940 & 4,858 & ,793 & $-8,79$ & 18,67 \\
\hline
\end{tabular}

En cuanto al nivel de intensidad del compromiso organizacional, la tabla 11 muestra que ICSA presenta un nivel alto de compromiso, en los institutos restantes se presenta un nivel de compromiso medio. 
Tabla 11. Nivel de intensidad de compromiso organizacional por instituto

\begin{tabular}{|l|l|l|l|l|l|}
\hline \multicolumn{7}{|c|}{ Instituto } & IIT & \\
\hline & ICSA & ICB & IADA & IT \\
\hline & Recuento & Recuento & Recuento & Recuento & \\
\hline \multirow{2}{*}{$\begin{array}{l}\text { Nivel de compromiso } \\
\text { organizacional }\end{array}$} & Bajo & 4 & 0 & 0 & 1 \\
\cline { 2 - 7 } & Medio & 33 & 23 & 16 & 14 \\
\cline { 2 - 7 } & Alto & 47 & 5 & 2 & 10 \\
\hline
\end{tabular}

Tabla 12. Nivel de intensidad de compromiso afectivo por instituto.

\begin{tabular}{|l|l|l|l|l|l|}
\hline \multicolumn{9}{|c|}{ Instituto } & IIT & \\
\hline & ICSA & ICB & IADA & IIT \\
\hline & Recuento & Recuento & Recuento & Recuento & \\
\hline \multirow{3}{*}{$\begin{array}{l}\text { Nivel de compromiso } \\
\text { afectivo }\end{array}$} & Bajo & 2 & 1 & 1 & 1 \\
\cline { 2 - 7 } & Medio & 26 & 18 & 10 & 12 \\
\cline { 2 - 7 } & Alto & 56 & 10 & 8 & 12 \\
\cline { 2 - 7 } & & & & & \\
\hline
\end{tabular}

Se encontró que los docentes que pertenecen al ICSA presentan un nivel de compromiso afectivo alto; en IADA e ICB el compromiso es medio, mientras que en IIT se da un empate entre el nivel medio y alto siendo solo en un caso el compromiso bajo.

Con respecto a la tabla de nivel de intensidad de compromiso de continuidad se puede observar que en todos los institutos generaron un nivel medio sobre esta dimensión. 
Tabla 13. Nivel de intensidad de compromiso de continuidad por instituto.

\begin{tabular}{|l|l|l|l|l|l|}
\hline & \multicolumn{5}{|c|}{ Instituto } \\
\hline \multirow{2}{*}{$\begin{array}{l}\text { Nivel de } \\
\text { compromiso de } \\
\text { continuidad }\end{array}$} & ICSA & ICB & IADA & IIT & \\
\cline { 2 - 7 } & Recuento & Recuento & Recuento & Recuento & \\
\cline { 2 - 7 } & Bajo & 6 & 3 & 1 & 5 \\
\cline { 2 - 7 } & Medio & 51 & 22 & 16 & 13 \\
\cline { 2 - 7 } & Alto & 27 & 3 & 2 & 7 \\
\hline
\end{tabular}

Tabla 14. Nivel de intensidad de compromiso normativo por instituto

\begin{tabular}{|c|c|c|c|c|c|}
\hline & \multicolumn{5}{|l|}{ Instituto } \\
\hline & ICSA & ICB & IADA & IIT & \\
\hline & Recuento & Recuento & Recuento & Recuento & \\
\hline \multirow{3}{*}{$\begin{array}{l}\text { Nivel de } \\
\text { compromiso } \\
\text { normativo }\end{array}$} & Bajo & 4 & 4 & 1 & 2 \\
\hline & Medio & 29 & 16 & 10 & 13 \\
\hline & Alto & 51 & 10 & 7 & 10 \\
\hline
\end{tabular}

Por último, la dimensión de compromiso normativo se presenta en nivel medio, siendo ICSA la excepción, ya que registra un nivel alto en esta dimensión según muestra la tabla 14.

\section{CONCLUSIONES}

lobjetivo de este traba$\boldsymbol{Y}$ jo consistió en conocer si existe diferencia significativa entre el compromiso organizacional y la percepción de apoyo entre los docentes en docentes que pertenecen a los institutos de ICSA e ICB.

Es posible que la diferencia en dos de las dimensiones se deba a distintos factores, en el caso de la dimensión afectiva podrían ser las experiencias positivas en el trabajo y las características personales del empleado. En el caso de la dimensión normativa estos factores podrían ser el grado de adopción de normas organizacionales, así como las inversiones hechas en favor de la organización debido al sentido de obligación que se ha generado (Meyer \& Allen, 1991).

En general, los docentes demostraron un nivel medio de compromiso hacia la Universidad Autónoma de Ciudad Juárez, siendo la dimensión que se refleja con mayor intensidad la afectiva, en menor intensidad la que corresponde a la dimensión de continuidad, lo cual refiere que los docentes con un alto compromiso afectivo permanecen laborando en la organización porque "desean hacerlo" en tanto que en la dimensión que se presentó con menor intensidad lo hacen porque "necesitan" hacerlo (Meyer \& Allen, 1991), estos resultados coinciden con los 
obtenidos por Barraza y Acosta (2008), Balfour Wechsler (1991), Tejada y Arias (2005), en los cuales se observó que el apego más fuerte hacia la organización es de tipo afectivo.

Por otra parte, es recomendable indagar por qué la dimensión de continuidad se presenta en nivel medio, ya que esta se asocia con la valoración que hace el empleado acerca del costo que implicaría dejar el empleo (De Frutos, Ruiz, \& San Martin, 1998), por lo tanto se asume que para los docentes el dejar la universidad no representa un costo alto considerando las inversiones hechas dentro de la institución, por lo que aumenta la posibilidad de que busquen otras alternativas de empleo (Dávila de León \& Jiménez García, 2014). Littlewood (2003) recomienda realizar una revisión del instrumento para medir el compromiso de continuidad, ya que los resultados de un metanálisi realizado por dicho autor muestran que posee baja confiabilidad, incluso sugiere que puede carecer de validez en nuestra cultura.

Estos resultados sugieren que es necesario realizar acciones encaminadas al aumento del compromiso organizacional de los docentes, debido a que presentan en general un nivel de compromiso medio. Sin embargo, lo ideal es contar con empleados altamente comprometidos para poder mantener la ventaja competitiva en la institución (Maldonado, Ramírez, García, \& Chairez, 2014). Este aspecto podría estar ligado a la percepción del apoyo organizacional, ya que se presenta de igual forma en un nivel medio, en estudios como el de Arias (2001), Tormo y Osca (2011) se encontró que la percepción del apoyo organizacional es un factor ligado al compromiso organizacional y que lo aumenta, por lo que es importante lograr que los docentes se sientan apoyados.

Con respecto al apoyo organizacional, no se encontraron diferencias con respecto a la percepción entre los grupos analizados, la percepción general que poseen encuentra en un nivel medio, sin embargo, puede aumentarse mejorando las condiciones de trabajo y recompensas, alentando el apoyo por parte de los superiores y la equidad o bien atendiendo a ciertas características de los empleados, como son la personalidad características demográficas (Rhoades \& Eisenberger, 2002).

\section{LIMITACIONES DEL ESTUDIO}

$\mathrm{D}$ ebe tomarse en cuenta que la muestra solicitada para obtener resultados significativos en la investigación no fue completada, siendo esta situación una limitante en este proyecto, sin embargo, la información obtenida es útil para dar a conocer que existe una diferencia significativa en el compromiso por parte de los docentes, en este sentido es recomendable completar o ampliar la muestra para lograr una representatividad más importante.

Una vía de estudio futuro podría consistir en investigar el por qué la dimensión de compromiso de continuidad se presenta en un nivel medio en los docentes y si difieren el compromiso organizacional y apoyo organizacional percibido por tipo de contrato del docente, ya que esta variable no fue considerada en el presente estudio. En este sentido, 


\section{7 novag}

Gakovik y Tetrick (2003) encontraron en su estudio que los empleados de tiempo parcial reportaron niveles más altos de $\mathrm{PAO}$, mientras que los empleados de tiempo completo reportaron mayores niveles de compromiso; sin embargo, no se encontraron diferencias significativas entre los dos grupos. Por otra parte Álvarez (2008) encontró que al incluir otras variables, trabajar a jornada parcial no conlleva a un menor compromiso de los empleados, por lo cual resultaría de interés incluir esta variable para su análisis.

\section{Bibliografía}

Álvarez, L. (2008). Determinantes del compromiso organizacional. ¿Están los trabajadores a tiempo parcial menos comprometidos que los trabajadores a tiempo completo? Cuadernos de Estudios Empresariales, 18, 73-88.

Arias, G. (Enero-Marzo de 2001). El compromiso personal hacia la organización y la intención de permanencia: algunos factores para su incremento. Contaduría y Administración (200), 5-12.

Ayensa, E., \& González, M. (2007). Definición,antecedentes y consecuencias del compromiso organizativo. Conocimiento, innovación y emprendedores: Camino al futuro, 3590-3609.

Balfour, D. L., \& Wechsler, B. (1991). Commitment, performance, and productivity in public organizations. Public Productivity \& Management Review, 14(4), 355-367.

Baran, B., Rhoades, S., \& Miller, L. (2012). Advancing organizational support theory into the twenty-first century world of work. J Bus Psychol, 27, 123-147.

Barraza, M. A., \& Acosta, C. M. (2008). Compromiso organizacional de los docentes de una institución de educación media superior. Innovación Educativa, 20-35.

Betanzos, D. N., Andrade, P. P., \& Paz, R. F. (2006). Compromiso organizacional en una muestra de trabajadores mexicanos. Revista de Psicología del Trabajo y de las Organizaciones, 22(1), 25-43.

Çağla, G. B. (2013). Examining organizational commitment of private school teachers. Journal of educational and instructional studies in the world, 3(2), 22-27.

Colakoglu, U., Culha, U., \& Atay, H. (Octubre de 2010). The effects of perceived otganisational support on employees affective outcomes: evidence from the hotel industry. Tourism and Hospitality Management, 16(2), 125-150.

Dávila de León, C., \& Jiménez García, G. (2014). Sentido de pertenencia y compromiso organizacional: predicción del bienestar. Revista de Psicología, 32(2), 272-302. 


\section{Rua}

De frutos, B., Ruiz, M., \& San Martin, R. (1998). Análisis factorial confirmatorio de las dimensiones del compromiso con la organización. Psicológica, 345-366.

Edel, R., García, A., \& Casiano, R. (2007). Clima y Compromiso Organizacional. Obtenido de Eumed: http://eumed.net/ libros/2007c/

Eisenberg, R., Armeli, S., Rexwinkel, B., Lynch, P., \& Rhoades, L. (2001). Reciprocation of perceived organizational support. Journal of applied psychology, 86(1), 42-51.

Eisenberger, R., Cummings, J., Armeli, S., \& Lynch, P. (1997). Perceived organizational support, discretionary treatment, and job satisfaction. Journal of applied psychology, 82(5), 812820.

Eisenberger, R., Huntington, R., Hutchison, S., \& Sowa, D. (1986). Perceived organizational support. Journal of Applied Psychology, 71(3), 500-507.

Florez, R. (2014). Clima laboral y compromiso organizacional en docentes universitarios. Tesis de grado de Licenciatura. Barquisimeto: Decanato Experimental de Humanidades y Artes, Universidad Centroccidental "Lisandro Alvarado".

Frutos, B., Ruiz, M. A., \& San Martin, R. (1998). Analisis factorial confirmatorio de las dimensiones del compromiso con la organizacion. Psicológica, 19, 345-366.

Gakovic, A., \& Tetrick, L. E. (Agosto de 2003). Perceived organizational support and work status: a comparison of the employment relationships of part-time and Full-time employees attending university classes. JSTOR, 24(5), 649-666.

García de Pilo, L., Useche, M. C., \& Schelesinger, M. W. (2013). Empowerment y compromiso organizacional docente investigador en el ámbito universitario Zuliano. Revista electrónica de bumanidades, educación y comunicación social, 6-19.

Gouldner, A. (1960). The norm of reciprocity: a preleminary statement. American Sociological Review, 25(2), 161-178.

Hernandez, M. Y., \& Guerrero, H. M. (Julio-Diciembre de 2006). Clima y compromiso a la organización de docentes de la facultad de enfermería de la Universidad Autónoma de San Luis Potosí. Conciencia Tecnológica(32), 0. 
Ko, J. W., Price, J. L., \& Mueller, C. W. (1997). Assessment of Meyer and Allen's three-component model of organizational commitment in South Korea. Journal of Applied Psychology, 82(6), 961-973.

Levinson, H. (1965). Reciprocation: the Relationship between man and organization. 370-390.

Littlewood, Z. H. (2003). Metanálisis del apoyo organizacional percibido y compromiso organizacional en organizaciones mexicanas. Revista interamericana de psicología ocupacional, 22(2), 45-46.

Maldonado, R. S., Guillen, J. A., \& Carranza, P. R. (2012). El compromiso organizacional del personal docente y administrativo de una universidad pública. Revista internacional administración y finanzas, 5(4), 135-142.

Maldonado, R. S., Ramírez, B. M., García, R. B., \& Chairez, V. A. (2014). Compromiso organizacional de los profesores de una universidad pública. Conciencia Tecnológica (47), 12-18.

Maldonado, R., Guillén, J. M., \& Carranza, P. E. (2011). El compromiso organizacional del personal administrativo en una universidad pública . Revista internacional administración y finanzas, 121-131.

Meyer, J. P., \& Allen, N. J. (1991). A three component conceptualization of organizational commitment. Human Resource Managment Review, 61-89.

Miranda, A. (2007). La Industria Automotriz en México. Instituto Polietécnico Nacional.

Moideenkutty, U., Blau, G., Kumar, R., \& Nalakath, A. (2001). Perceived organizational support as a mediator of the relationship of perceived situational factors to affective organizational commitment. Applied psychology: an international review, 615-634.

Mowday, R. T., Steers, R. M., \& Porter, L. W. (1979). The Measurement of Organizational Commitment. Journal of Vocational Behavior, 14, 224-247.

Müggenburg, R., \& Pérez, C. (Enero-Abril de 2007). Tipos de estudio en el enfoque de investigación cuantitativa. Revista Enfermería Universitaria ENEO-UNAM, 4(1), 35-38. 
Porter, L. W., Steers, R. M., Mowday, R. T., \& Boulian, P. V. (1974). Organizational commitment, job satisfaction and turnover among psychiatric technicians. Journal of Applied Psychology, 59(5), 603-609.

Rhoades, L., \& Eisenberger, R. (2002). Perceived organizational support: a review of literature. Journal of Applied Psychology, 87(4), 698-714.

Robbins, S. P., \& Judge, T. A. (2009). Comportamiento Organizacional. México : Perason Education .

Robles, H., \& De la garza, C. (2009). El compromiso organizacional y la actitud de os profesores de ciencias económico administrativas de las IES públicas frente a las necesidades de capacitación institucionales. Ciencia@UAQ,2(2),69-78.

Ruiz de Alba, R. J. (2013). El compromiso organizacional: un valor personal y empresarial en el marketing interno. Revista de estudios empresariales, 2(1), 67-86.

Shumaila, S., Aslam, R., Sadaqat, S., Maqsood, S., \& Sadaf, N. (2012). Perceived organizational support as a predictor of organizational commitment: a comparative study on public and private sector nurses. Proceedings of 2 nd International Conference on Business Management.

Sivalogathasan, V., \& Ashmel, H. (2014). Changes in employeremployee relationship: impact of perceived organizational support on social exchange of the outsourcing industry in Sri Lanka. Skyline Business Journal, 9(1), 43-49.

Soberanes, R., \& De la Fuente, I. (2009). El clima y el compromiso organizacional en las organizaciones. Revista internacional la nueva gestión organizacional, 5(9), 120-127.

Tejada, T. M., \& Arias, G. (2005). Prácticas organizacionales y el compromiso de los trabajadores hacia la organización. Enseñanza e Investigación en Psicologia, 10(2), 295-309.

Tormo, C., \& Osca, S. (2011). Antecedentes organizacionales y personales de las Intenciones de compartir conocimiento: apoyo, clima y compromiso con la organización. Revista de psicología del trabajo y las organizaciones, 213-226. 


\section{1 novag \\ कृ}

Torres, S., \& Díaz, F. (2012). Compromiso organizacional: actitud laboral asertiva para la competitividad de las organizaciones. Revista de formación gerencial, 11(1), 80-101.

Wu, C.-C., \& Liu, N.-T. (2014). Perceived organizational support,organizational commitment and service-oriented organizational citizenship behaviors. International Journal of Business and Information, 61-88.

Yasar, M. F., Emhan, A., \& Ebere, P. (2014). Analysis of organizational justice, supervisor support, and organizational commitment. Journal of Business Studies Quarterly, 38-46.

Zamora, P. G. (2009). Compromisos organizacionales de los profesores chilenos y su relación con la intención de permanecer en sus escuelas. Revista Latinoamericana de Psicologia, 41(3), 445-460. 